\title{
Millisecond Pulsar Surveys
}

\author{
D. C. Backer \\ Astronomy Department and Radio Astronomy Laboratory \\ University of California \\ Berkeley, CA 94720
}

\begin{abstract}
In 1982 a new class of pulsars was defined by the discovery of a star with a millisecond rotation period, $1.6 \mathrm{~ms}$. In the past 3.5 years two additional pulsars with millisecond periods have been discovered. The rapid spin of these pulsars is attributed to mass transfer in a low-mass binary progenitor system. This hypothesis is supported by the presence of companions in two of the three millisecond pulsars. These recent discoveries have led both to a deeper understanding of the final stages of stellar evolution in binary systems, and to closer ties between the observational study of neutron stars by radio, optical and X-ray techniques. In addition the millisecond pulsars provide precise astrophysical clocks that can be used to improve the solarsystem ephemeredes and to search for a background of gravitational waves that may have been produced in the early stages of the visible universe. Old and ongoing searches for new millisecond pulsars are described in this paper.
\end{abstract}

\section{INTRODUCTION}

Three millisecond pulsars are presently known. Their periods range from 1.6 to $6.1 \mathrm{~ms}$. Although the periods of all three objects are short, their ages probably exceed one million years since there is no sign of debris in their vicinity, and, in the case of the nearest one, the companion star is cool. In sharp contrast the next fastest pulsar, the Crab pulsar, is located in the midst of a brilliant nebula and is known from Chinese court records to have an age of 932 years (Wang 1987). Two of three millisecond pulsars have low-mass companions with orbital periods of 12 and 120 days. Consideration of the of low-mass binary evolution (van den Heuvel $198 \%$ and references therein) provides a solid framework for understanding the existence of old pulsars with rapid rotation periods. Both observational evidence and theoretical discussions lend support to the identification of a subclass of pulsars - those with periods less than about 10 milliseconds. 
Further progress in the understanding of the origin and evolution of fast pulsars will require deeper and more complete surveys than are presently available. Upon completion these surveys must be carefully analyzed to define the galactic population of fast pulsars following the early discussion by Gunn and Ostriker (1970) and the recent analysis by Lyne, Manchester and Taylor (1985). In the case of binary systems important information on the evolution of the system can be obtained from optical detection (or limits) of the companion as shown recently by Kulkarni (1986).

New millisecond pulsars will be important for the application of pulsar observations to other branches of science: timekeeping, solar system dynamics, celestial astrometry, interstellar medium, general relativity, and cosmology. Intense studies of the present millisecond pulsars have already contributed to these other subjects and new objects will certainly lead to further developments. In some areas new objects anywhere in space would do as long as they are bright; in other areas, such as timekeeping and cosmology, a distribution on the sky is needed. In all cases a survey needs only to be successful in new discoveries, since completeness is less important.

One area of inquiry was neglected in the preceding discussion: neutron star structure. This deserves special mention in this Symposium. Many of the interesting applications of millisecond pulsars are dependent on the stability of the rotation of the microwave beams attached to these neutron stars. While models of pulsar emission are still schematic, it is clear that stability of the beam rotation implies stability of the rotation of the neutron star to which it is attached; the reverse conclusion cannot be made with certainty. Despite the rapid rotation period of the 1.6-ms pulsar its observed rotation is extremely stable (Davis et al. 1985). This is remarkable since the neutron star has a considerable equatorial bulge which must decrease continually as the star spins down. If a 'glitch' were to appear in one of the millisecond pulsars, we would lose its use as a precise clock, but gain valuable insight into the structure of a neutron star in rapid rotation.

The three known millisecond pulsars were discovered by three different paths. The first, $1937+21$, can be traced back to a peculiar complex of radio sources in the $4 \mathrm{C}$ catalog, $4 \mathrm{C} 21.53$ (Backer et al. 1982; Backer 1984). The second, 1953+29, was discovered by Boriakoff et al. (1983) by seaching one square degree fields centered on gamma ray source positions. The association of this pulsar with the target gamma ray source is questionable. If $1953+29$ is not the gamma ray source, then the Boriakoff et al. search reduces to a deep general search of about four square degrees near the galactic plane. The most recent millisecond pulsar, 1855+09, was the result of a rapid survey of 800 square degrees along portion of the galactic plane that is visible with the 
sensitive Arecibo telescope (Seigelstein et al. 1986). The properties of these three pulsars are summarized in Table 1.

\begin{tabular}{cccccc}
\multicolumn{5}{c}{ Table 1 } \\
PSR & $\begin{array}{c}\text { Rotation } \\
\text { Period } \\
(\mathrm{ms})\end{array}$ & $\begin{array}{c}\text { Period } \\
\text { Derivative } \\
\left({ }^{*} 10^{18}\right)\end{array}$ & $\begin{array}{c}\text { Orbital } \\
\text { Period } \\
(\mathrm{d})\end{array}$ & $\begin{array}{c}\text { Companion } \\
\left(\mathrm{M}_{0}\right)\end{array}$ & $\begin{array}{c}\text { Dispersion } \\
\text { Measure } \\
\left(\mathrm{pc} \mathrm{cm}{ }^{-3}\right)\end{array}$ \\
& & & & & \\
$1855+09$ & 5.436 & 0.16 & 12 & $0.3-0.4$ & 13 \\
$1937+21$ & 1.558 & 0.11 & - & - & 71 \\
$1953+29$ & 6.133 & 0.03 & 120 & $0.2-0.4$ & 105
\end{tabular}

\section{SEARCH STRATEGIES}

Pulsars have small radio luminosities, $10^{30} \mathrm{erg} \mathrm{s}^{-1}$, with logarithmic frequency indices steeper than -1 , and are distributed throughout the galaxy with a scale height of $400 \mathrm{pc}$. As a result surveys for new pulsars would be most sensitive if conducted at low frequencies with wide bandwidths. The integration time would be as long as possible consistent with the need to cover the independent beam areas on the sky in a given amount of telescope time.

However the bandwidth is restricted by the dispersion of the signal resulting from interstellar plasma. In most surveys sensitivity is enhanced by sampling many channels whose bandwidth is proportional to the cube of the observing frequency and the maximum ratio of dispersion measure to period expected. The penalty one pays for multi-channel sampling is that the pulse search for each beam position requires a two-dimensional algorithm for which the computations increase with the square of the minimum period if the total bandwidth and maximum dispersion measure are fixed.

If the computational power of a search is fixed, then searches are nearly frequency independent: the loss of sensitivity with increasing frequency from the steep source spectrum is compensated by the increased allowed bandwidth per channel that results from reduced dispersion. Another important factor that contributes to the frequency insensitivity is the decrease in background noise from galactic synchrotron radiation as frequency increases. The penalty here is that as the frequency increases the beam solid angle decreases, and more observing time is required to cover the same search solid angle.

The discovery of millisecond pulsars has brought attention to yet another factor that limits pulsar surveys - the broadening of pulses that results 
from multipath propagation in the interstellar medium. An impulse of radiation from the star is received at the Earth as an exponential-shaped pulse with decay time of $\tau_{s}$ which varies with the fourth power of the radio wavelength and is a steep function of the dispersion measure. Figure 1 presents measured values of $\tau_{s}$ scaled to an observing frequency of $1 \mathrm{GHz}$ as a function of the dispersion measure. The median broadening time between the plotted lines can be expressed as a function of the dispersion measure as follows:

$$
\begin{aligned}
\tau_{s} & =10^{7.25} \mathrm{~ms} \nu^{-4} D M^{2}, 0<D M<125 \mathrm{pc} \mathrm{cm}^{-3} \\
& =10^{-10} \mathrm{~ms} \nu^{-4} D M^{4}, 125<D M<1000 \mathrm{pc} \mathrm{cm}^{-3}
\end{aligned}
$$

As shown in Figure 1 individual objects can differ from the median by an order of magnitude in both directions. This figure clearly shows that surveys for millisecond pulsars out to modest dispersion measures must choose an observing frequency near, or above, $1 \mathrm{GHz}$. Search algorithms look for as many as 16 harmonics of the pulsar frequency, and it is the highest harmonic, or smallest fraction of the period, desired that must not be smeared by interstellar scattering. The survey by Clifton and Lyne (1986) at Jodrell Bank using 1.4 GHz provides an example of the importance of higher observing frequency. The higher frequency does not limit the detection capability for comparable observing time, as discussed above. However the beam solid angle is reduced and as a result the time required to complete a survey is increased.

The detectability of a millisecond pulsar in a fast binary system can be limited by the Doppler acceleration during an integration time. If we consider a circular orbit for a binary of two $1.4 M_{o}$ neutron stars, then there is a limiting product of rotational period and orbital period below which the signal will be Doppler spread in a Fourier analysis. Figure 2 shows the known binary pulsars in a rotation period-binary period diagram. Three limiting lines are displayed for integration times of 100,300 and $1000 \mathrm{~s}$. These lines were drawn under the assumption that the fourth harmonic of the pulsar rotation frequency is smeared by the inverse of the integration time. The most interesting binary $1913+16$ is within the region where existing surveys are compromised by Doppler acceleration during the integration. Similar limits apply to the low-mass binary companion case. The three lines in Figure 2 correspond to integration times of approximately 30,100 and $300 \mathrm{~s}$, respectively, for the low-mass case. In principle an algorithm could be devised to search in acceleration along with period and dispersion. To date no survey has explored this domain. 


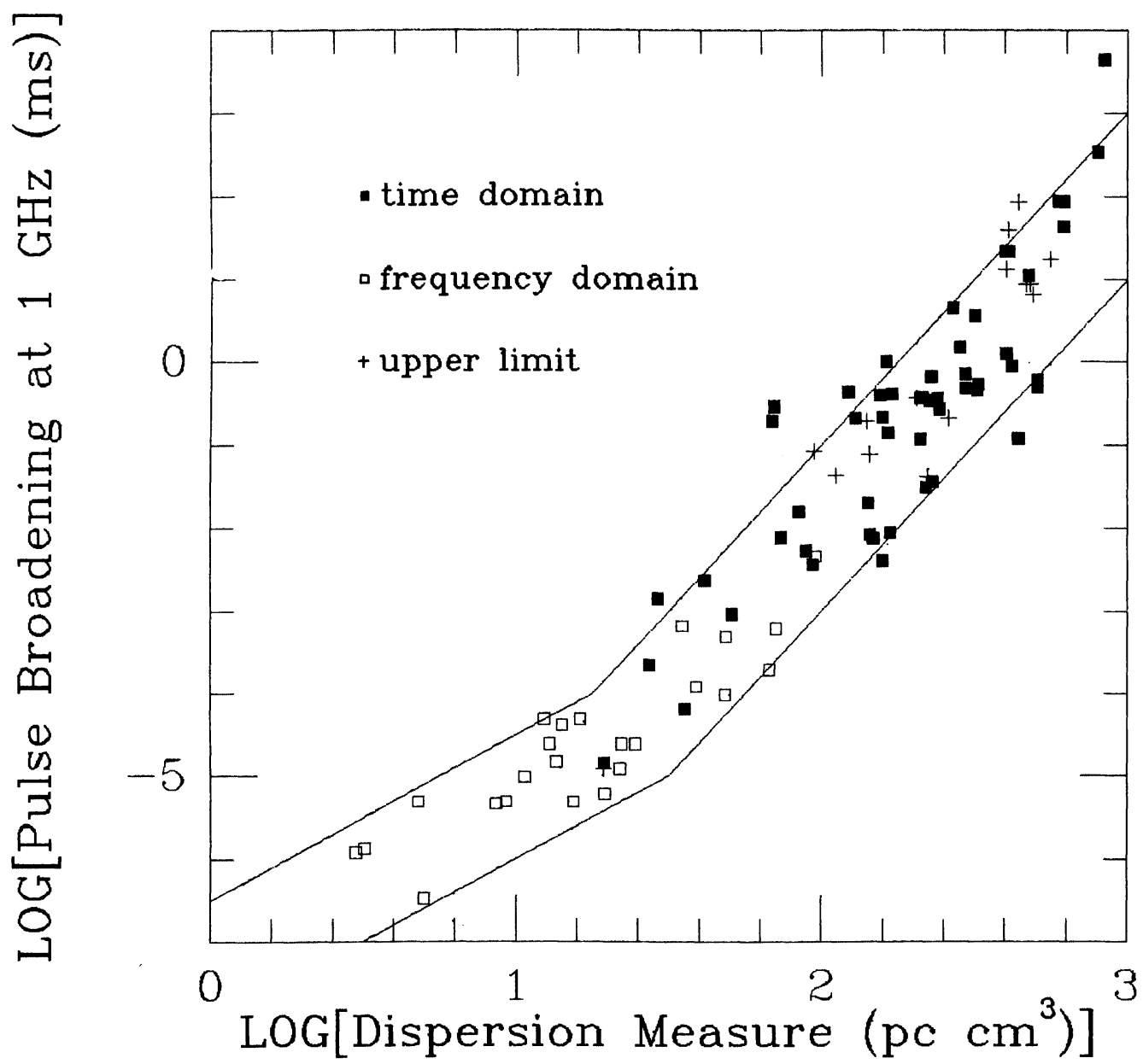

Figure 1 Pulse broadening by interstellar scattering as a function of the dispersion measure at $1 \mathrm{GHz}$ observing frequency. Open squares are from measures of the decorrelation bandwidth and filled squares are from direct deconvolution of average pulse profiles. Plus symbols are upper limits to scattering time. 
The difficulties in surveying the sky for millisecond pulsars discussed above led a number of investigators to try continuum techniques for identifying millisecond pulsar candidates. Several continuum techniques were suggested by aspects of the discovery story of $1937+21$. One peculiarity of 4C21.53 was its small size indicated by interplanetary scintillation in spite of its low galactic latitude. Purvis et al. (1984) pursued other low-latitude 'scintars' without success. The steep meter-wavelength spectrum of $4 \mathrm{C} 21.53$ was also peculiar, and other compact sources with steep spectra have been identified by Erickson. While one or two of these remain interesting, no pulsars have been identified. Interstellar scintillation was observed from 1937+21 before it was known to be a millisecond pulsar. As a result a rapid survey of 3400 square degrees was conducted by Stevens et al. (1984) to identify scintillating sources that might be fast pulsars. Again they found none. The strong linear polarization of $1937+21$ was early evidence in favor of its pulsar identification. Several surveys have now been conducted trying to identify at long wavelengths compact and highly polarized sources for subsequent pulse searches. Stevens (1986) has used the Westerbork array at $327 \mathrm{MHz}$ to identify pulsar candidates, and then the Arecibo telescope to search for pulses. Lyne (1986) has used Jodrell Bank instruments at $408 \mathrm{MHz}$ for a similar search. Neither search has led to new pulsars. A search for compact sources at the centers of globular clusters was conducted by Hamilton, Helfand and Becker (1985) based on the proposed connection between millisecond pulsars and the low-mass Xray binaries. One source in M28 remains as a potential hidden pulsar that is too weak or too scattered for present pulse detection techniques.

\section{NEW MILLISECOND PULSAR SURVEYS}

The difficult task of sifting through the multidimensional haystack of possibilities for millisecond pulsars - two sky coordinates, dispersion measure (distance) and period - has just begun. The first surveys that have reached the sensitivity capable of detecting a significant galactic population of millisesand nulcars are summarized in Table 2 below. The next year or so will lead to much firmer evidence on the number and possibly the distribution of these exotic stars. 
Table 2

Millisecond Pulsar Surveys

\begin{tabular}{|c|c|c|c|c|c|}
\hline Investigators & $\begin{array}{c}\text { Telescope } \\
(\mathrm{MHz})\end{array}$ & $\begin{array}{l}\text { Frequency } \\
\text { Time (ms) }\end{array}$ & $\begin{array}{l}\text { Sampling } \\
\text { Angle* }\end{array}$ & $\begin{array}{l}\text { Solid } \\
\text { Time (s) }\end{array}$ & Integration \\
\hline Seigelstein \& & Arecibo & 430 & 0.3 & $10 \times 30$ & 39 \\
\hline
\end{tabular}

Taylor

Manchester \&

Molonglo

843

0.5

$2 \times 105$

130

D'Amico \&

Taylor

Lyne

Jodrell Bank

610

0.3

$20 \times 110$

79

1400

0.3

$2 \times 110$

79

Backer \& Green Bank

825

0.25

$20 \times 150$

32

Kulkarni \& Deep Space Net

2300

0.5

$2 \times 100$

64

Clifton

* sky coverage listed in degrees of latitude by degrees of longitude.

\section{REFERENCES}

Backer, D. C., Kulkarni, S. R., Heiles, C., Davis, M. M., and Goss, W. M. 1982, Nature, $300,615$.

Backer, D. C. 1984, J. Astron. Astrophys., 5, 187.

Hamilton, T. T., Helfand, D. J., and Becker, R. H. 1985, Astron. J., 90, 606.

Boriakoff, V., Buccheri, R., and Fauci, F. 1983, Nature, 304, 417.

Clifton, T. R., and Lyne, A. G. 1986, Nature, 320, 43.

Davis, M. M., Taylor, J. H., Weisberg, J. M., and Backer, D. C. 1985, Nature, 315, 547.

Gunn, J. E., and Ostriker, J. P. 1970, Ap. J., 160, 979.

Kulkarni, S. 1986, preprint.

Lyne, A. G. 1986, personal communication.

Lyne, A., Manchester, R. N., and Taylor, J. H. 1985, M.N.R.A.S., 213, 613.

Purvis, A., et al. 1984, NRAO Workshop on 'Millisecond Pulsars', edited by S. P. Reynolds and D. R. Stinebring, p. 252. 
Seigelstein, D.J., et al., 1986, Nature, in press.

Stevens, M. et al. 1984, NRAO Workshop on 'Millisecond Pulsars', edited by S. P. Reynolds and D. R. Stinebring, p. 242.

Stevens 1986, Ph. D. thesis, University of California, Berkeley.

Wang, Z. R. 198 , IAU Symposium 125, this volume.

van den Heuvel, E. P. J. 1985, IAU Symposium, this volume.

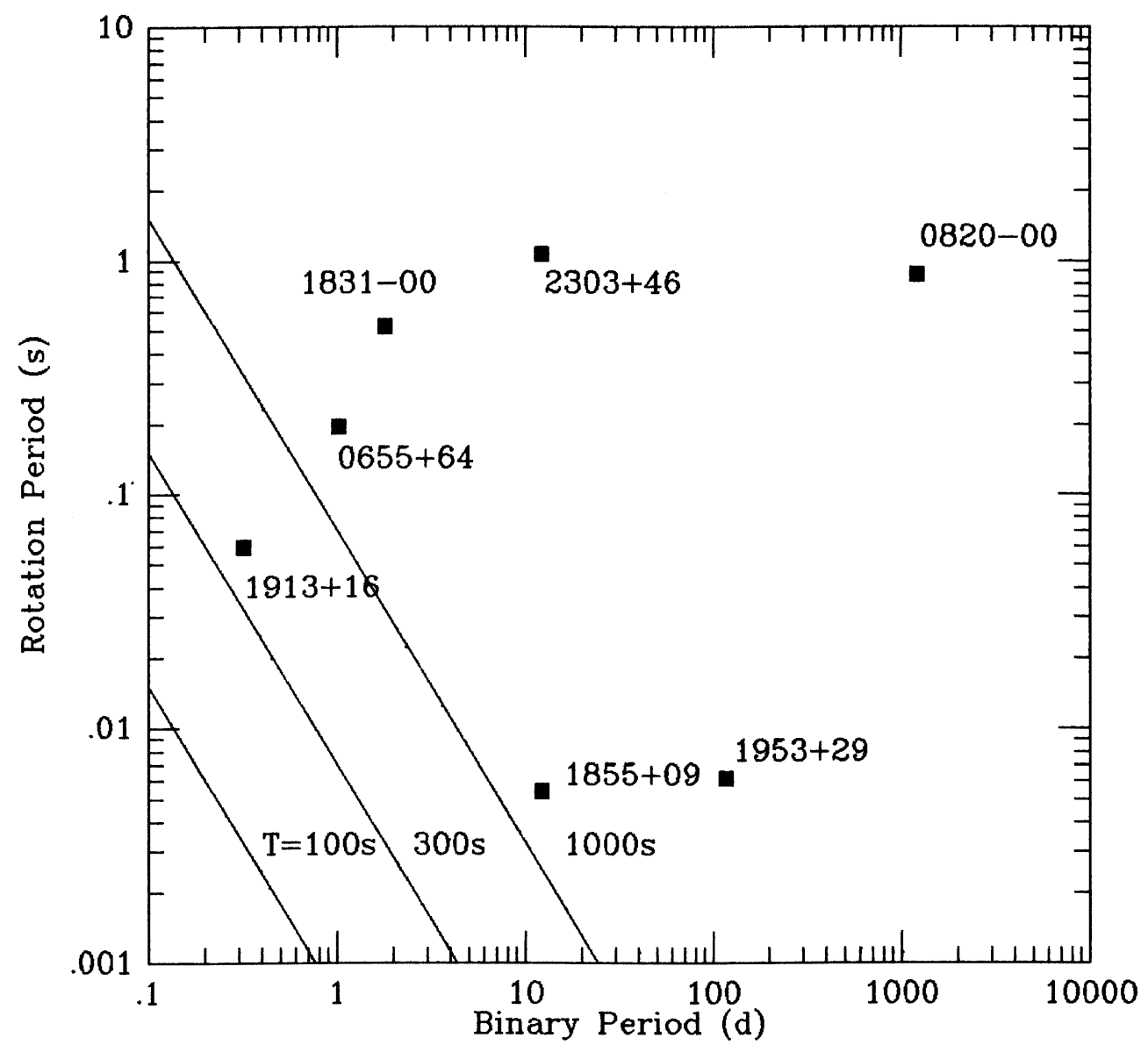

Figure 2 Binary pulsars in a rotation period - orbital period diagram. Slanting lines in lower left corner represent upper limits to parameters of pulsars detectable with integration times marked under the assumptions discussed in the text. 


\section{DISCUSSION}

S. Rulkarni: Regardng your point about loss of sensitivity towards fast, tight binaries I would like to just say that in principle one can circumvent the loss in sensitivity by including a variety of acceleration either before the Fourier transform or after it. Probably the increase in computing is no more than a factor of 10 .

D. Backer: I agree that there are direct methods for adding a search in acceleration to the search in period, dispersion and celestial position. 